\title{
Blood pressure level and risk of major cardiovascular events and all-cause of mortality in patients with type 2 diabetes and renal impairment: an observational study from the Swedish National Diabetes Register
}

\author{
Hanri Afghahi • Maria K. Svensson • \\ Mirnabi Pirouzifard • Björn Eliasson • \\ Ann-Marie Svensson
}

Received: 17 September 2014 / Accepted: 12 February 2015 /Published online: 14 March 2015

(C) Springer-Verlag Berlin Heidelberg 2015

\begin{abstract}
Aims/hypothesis We assessed the relationship between BP and risk of cardiovascular events (CVEs) and all-cause mortality in patients with type 2 diabetes and renal impairment (estimated GFR $<60 \mathrm{ml} \mathrm{min}{ }^{-1} 1.73 \mathrm{~m}^{-2}$ ) treated in clinical practice.

Methods A total of 33,356 patients (aged $75 \pm 9$ years, diabetes duration of $10 \pm 8$ years) with at least one serum creatinine and BP value available in the Swedish National Diabetes Register between 2005 and 2007 were followed up until 2011 or death. The relationships between mean BPs, CVEs and all-cause mortality were examined using time-dependent Cox models to estimate HRs, adjusting for cardiovascular risk factors and ongoing medications.

Results During the follow-up period (mean 5.3 years), 11,317 CVEs and 10,738 deaths occurred. The lowest risks of CVEs and all-cause mortality were observed with a systolic BP (SBP) of 135-139 and a diastolic BP (DBP) of 72-74 mmHg, and the
\end{abstract}

Hanri Afghahi and Maria K. Svensson contributed equally to this work.

Electronic supplementary material The online version of this article (doi:10.1007/s00125-015-3548-1) contains peer-reviewed but unedited supplementary material, which is available to authorised users.

\section{H. Afghahi}

Department of Nephrology, Skaraborgs Sjukhus, Skövde, Sweden

M. K. Svensson $(\varangle) \cdot$ B. Eliasson

Department of Medicine, Sahlgrenska University Hospital,

SE-413 45 Gothenburg, Sweden

e-mail: maria.svensson3@gu.se

M. Pirouzifard · A.-M. Svensson

Center of Registers in Region Västra Götaland, Gothenburg, Sweden highest risks were observed for those with SBP intervals 80 120 (CVE HR 2.3 [95\% CI 2.0, 2.6] and all-cause mortality HR 2.4, [95\% CI 2.1, 2.7]) and 160-230 mmHg (CVE HR 3.0 $[95 \%$ CI $2.6,3.3]$ and all-cause mortality HR $2.0[95 \%$ CI 1.8 2.3]) and DBP intervals 40-63 mmHg (CVE HR 2.0 [95\% CI $1.8,2.2]$, all-cause mortality HR 2.0 [95\% CI 1.8, 2.2]) and 83-125 mmHg (CVE HR 2.3 [95\% CI 2.0, 2.5], all-cause mortality HR 2.3 [95\% CI 2.0, 2.6]).

Conclusions/interpretation In this nationwide cohort of patients with type 2 diabetes and renal impairment, the risk of CVEs and all-cause mortality increased significantly with both high and low BPs, while an SBP of 135-139 mmHg and DBP of $72-74 \mathrm{mmHg}$ were associated with the lowest risks of CVEs and death.

Keywords Blood pressure - Cardiovascular disease . Epidemiology and outcomes $\cdot$ Renal function $\cdot$ Type 2 diabetes 


\section{Introduction}

Hypertension is present in $20-55 \%$ of patients at the time of diagnosis of type 2 diabetes [1]. Elevated BP is associated with an increase in the risk of cardiovascular disease (CVD) and death in the general population [2-5]; when hypertension is combined with diabetes, the CVD risk increases further [6]. Previous studies have shown that in patients with type 2 diabetes a systolic BP (SBP) of $140 \mathrm{mmHg}$ or higher increases the risk of CHD, stroke, cardiovascular events (CVEs) and allcause mortality [7-10]. In addition, other studies have shown an increase in the risk of CVEs and mortality when SBP is reduced to below $120 \mathrm{mmHg}[11,12]$. A diastolic BP (DBP) of $<70 \mathrm{mmHg}$ at baseline and during follow-up has been associated with a significantly increased risk of CVD [13].

Based on these studies, a hypothesis of a J-shaped relationship between treated BP and the risk of CVEs and mortality has been proposed and is still under debate $[7,14]$. The current treatment guidelines by the ADA and EASD, however, recommend a BP target in patients with diabetes of 140/ $90 \mathrm{mmHg}$ unless signs of end-organ damage such as albuminuria and retinopathy are present; if so, a stricter BP target of $130 / 80 \mathrm{mmHg}$ is recommended [15]. In patients with renal impairment (RI), the Kidney Disease: Improving Global Outcomes workgroup recommend a BP of $<140 / 90 \mathrm{mmHg}$ in the absence of albuminuria and $<130 / 80 \mathrm{mmHg}$ in the presence of albuminuria to reduce the risk of CVD. In addition, the guidelines mention that other factors such as age and cardiovascular comorbidities should be considered [16].

Optimal BP levels in high risk patients have thus not yet been established. The aim of this study was therefore to assess the relationship between BP level and the risk of cardiovascular events and all-cause mortality in a nationwide observational study of unselected patients with type 2 diabetes and RI who were treated in clinical practice and reported to the Swedish National Diabetes Register (NDR).

\section{Patients and methods}

The NDR The NDR was initiated in 1996. Information is collected at least once yearly during patient visits and reported to the NDR. All patients provide informed consent to be registered before inclusion, and all information is stored in a central database. The Regional Ethics Review Board at the University of Gothenburg approved the study, which was performed in accordance with the Declaration of Helsinki.

We included 33,356 patients with type 2 diabetes and RI (estimated GFR [eGFR] of $<60 \mathrm{ml} \mathrm{min}^{-1} 1.73 \mathrm{~m}^{-2}$ ). Inclusion criteria were a diagnosis of type 2 diabetes with a reported serum creatinine value between $>20$ and $<800 \mu \mathrm{mol} / 1$ at baseline. Reported type 2 diabetes was defined as patients having a reported treatment with diet only or with oral glucose- lowering agents only, or an onset of diabetes at 40 years or older and treated with insulin either alone or combined with oral glucose-lowering agents. Patients with an extreme body composition, i.e. BMI $\leq 18$ or $\geq 45 \mathrm{~kg} / \mathrm{m}^{2}$ ( $\left.n=359\right)$ were excluded. In addition, patients with severe RI (eGFR $<15 \mathrm{ml} \mathrm{min}{ }^{-1} 1.73 \mathrm{~m}^{-2} ; n=274$ ) were excluded because reporting of this patient group to the NDR is limited and highly selective. The clinical characteristics of patients with type 2 diabetes fulfilling the same inclusion criteria but with normal renal function $(n=117,947)$ are given in electronic supplementary material (ESM) Table 1 for comparison.

Examinations at baseline BP was determined as the mean value of the reported BPs from baseline to an endpoint or the end of the study (usually with annual reporting). All BPs used in this study are the BPs reported to the NDR, and according to the instructions they should be taken according to Swedish standard for BP recording. The Swedish standard for BP recording is the mean value ( $\mathrm{mmHg}$ ) of two supine readings (Korotkoff I-V) with a cuff of an appropriate size, after at least $5 \mathrm{~min}$ of rest.

Analyses of serum creatinine, $\mathrm{HbA}_{1 \mathrm{c}}$ and blood lipids were performed at local laboratories. Renal function expressed as the eGFR (in millilitres per minute per $1.73 \mathrm{~m}^{2}$ ) was calculated using the Modification of Diet in Renal Disease study equation [17]. Albuminuria was defined as micro- or macroalbuminuria, i.e. a urinary AER of 20-200 or $>200 \mu \mathrm{g} / \mathrm{min}$ in two out of three consecutive tests at baseline. A smoker was defined as a patient who smoked one or more cigarette per day or used a pipe, or who had stopped smoking within the past 3 months.

Follow-up and definition of endpoints Patients were followed from the baseline examination (between 1 July 2005 and 31 December 2007) until a first CVE (primary endpoint) and/or death, or otherwise until 31 December 2011. The mean follow-up time was 5.3 years. A CVE was defined as CHD, stroke or peripheral arterial disease (PAD), whichever came first, and the definition of CHD was myocardial infarction (MI) (ICD-10 code I21; www.who.int/classifications/icd/en/), unstable angina (ICD-10 code I20.0), percutaneous coronary intervention and/or coronary artery bypass grafting. Fatal CHD was defined as ICD-10 codes I20-I25, stroke was defined as non-fatal or fatal cerebral infarction, intracerebral haemorrhage or unspecified stroke (ICD-10 codes I61, I63, I64 and I67.9), PAD (ICD-10 codes I70.2, I73. 1, I73.9 and I79.2) and congestive heart failure (ICD-10 code I50) [18, 19]. Data on all events were retrieved by data linkage with the Swedish Cause of Death and the Hospital Discharge Registers (National Board of Health and Welfare, Sweden), which is a reliable validated alternative to revised hospital discharge records and death certificates [20, 21]. 
Table 1 Baseline clinical and biochemical characteristics in patients with type 2 diabetes and $\mathrm{RI}^{\mathrm{a}}$ with and without albuminuria ${ }^{\mathrm{b}}$
Data are means $\pm \mathrm{SD}$ or frequencies $(\%)$; missing data for triacylglycerol and HDL-cholesterol $(n=11,097) ;$ LDL-cholesterol $(n=$ $7,749) ; \mathrm{HbA}_{1 \mathrm{c}}(n=276)$; lipidlowering medication $(n=1,437)$; smokers $(n=2,556)$; CVD represents CHD and/or stroke

${ }^{\mathrm{a}}$ eGFR $<60 \mathrm{ml} \mathrm{min}^{-1} 1.73 \mathrm{~m}^{-2}$

${ }^{\mathrm{b}}$ Mean follow-up time 5.3 years

${ }^{c}$ eGFR according to the Modification of Diet in Renal Disease study equation

${ }^{\mathrm{d}}$ Urinary AER 20-200 mg/min

${ }^{\mathrm{e}}$ Urinary AER > $200 \mathrm{mg} / \mathrm{min}$

\begin{tabular}{|c|c|c|c|}
\hline Variables & $\begin{array}{l}\text { RI without albuminuria } \\
(n=23,441)\end{array}$ & $\begin{array}{l}\text { RI with albuminuria } \\
(n=9,915)\end{array}$ & $p$ value \\
\hline Age (years) & $75 \pm 9$ & $75 \pm 9$ & 0.003 \\
\hline Diabetes duration (years) & $9 \pm 8$ & $12 \pm 8$ & $<0.0001$ \\
\hline $\mathrm{HbA}_{1 \mathrm{c}}(\mathrm{mmol} / \mathrm{mol})$ & $52 \pm 11$ & $56 \pm 13$ & $<0.0001$ \\
\hline $\mathrm{HbA}_{1 \mathrm{c}}(\%)$ & $6.9 \pm 1.2$ & $7.3 \pm 1.1$ & $<0.0001$ \\
\hline $\mathrm{SBP}(\mathrm{mmHg})$ & $140 \pm 18$ & $143 \pm 20$ & $<0.0001$ \\
\hline DBP (mmHg) & $74 \pm 10$ & $75 \pm 10$ & $<0.0001$ \\
\hline BMI $\left(\mathrm{kg} / \mathrm{m}^{2}\right)$ & $29 \pm 5$ & $29 \pm 5$ & $<0.0001$ \\
\hline Total cholesterol (mmol/l) & $4.9 \pm 1.1$ & $4.8 \pm 1.0$ & $<0.0001$ \\
\hline LDL-cholesterol (mmol/l) & $2.7 \pm 0.9$ & $2.6 \pm 1.0$ & $<0.0001$ \\
\hline HDL-cholesterol (mmol/l) & $1.3 \pm 0.4$ & $1.4 \pm 0.4$ & $<0.0001$ \\
\hline Triacylglycerol (mmol/l) & $1.9 \pm 1.1$ & $2.1 \pm 1.3$ & $<0.0001$ \\
\hline Serum creatinine $(\mu \mathrm{mol} / \mathrm{l})$ & $108 \pm 23$ & $130 \pm 40$ & $<0.0001$ \\
\hline eGFR $\left(\mathrm{ml} \mathrm{min} \min ^{-1} 1.73 \mathrm{~m}^{-2}\right)^{\mathrm{c}}$ & $49 \pm 8$ & $45 \pm 11$ & $<0.0001$ \\
\hline Male (\%) & 36 & 59 & $<0.0001$ \\
\hline Smokers (\%) & 7 & 10 & $<0.0001$ \\
\hline Any retinopathy (\%) & 28 & 48 & $<0.0001$ \\
\hline History of CVD (\%) & 29 & 37 & $<0.0001$ \\
\hline History of CHF (\%) & 13 & 18 & $<0.0001$ \\
\hline \multicolumn{4}{|l|}{ Diabetes treatment } \\
\hline Diet only $(\%)$ & 29 & 16 & $<0.0001$ \\
\hline Oral glucose-lowering drug (\%) & 37 & 33 & $<0.0001$ \\
\hline Insulin+oral glucose-lowering drug (\%) & 15 & 22 & $<0.0001$ \\
\hline Insulin only (\%) & 19 & 29 & $<0.0001$ \\
\hline Lipid-lowering drug (\%) & 54 & 58 & $<0.0001$ \\
\hline Antihypertensive drug (\%) & 86 & 89 & $<0.0001$ \\
\hline Microalbuminuria $^{\mathrm{d}}(\%)$ & - & 60 & \\
\hline Macroalbuminuria ${ }^{\mathrm{e}}(\%)$ & - & 40 & \\
\hline
\end{tabular}

In order to analyse non-linear relationships, we included both SBP and the squares of SBP for CVE (1-survival rate) in the Cox model; the same analyses were performed for DBP. We also created splines with nine knots at the decile using the Transreg procedure (Figs 1, 2). All statistical analyses were performed in SAS V. 9.3 (SAS Institute, Cary, NC, USA) [22].

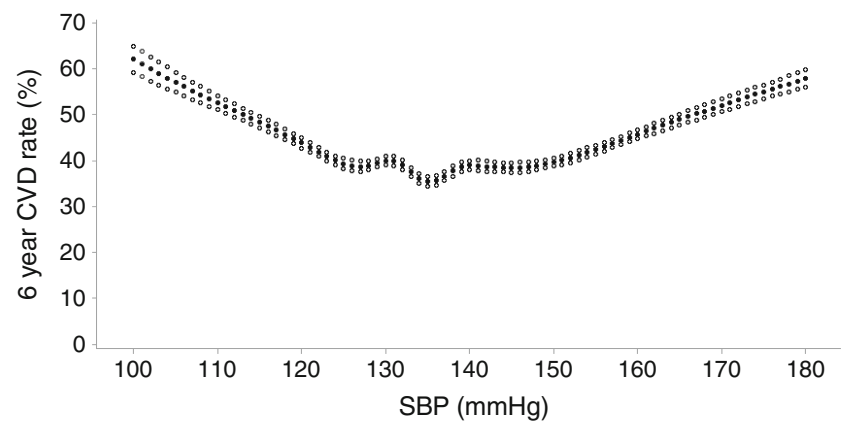

Fig. 1 Spline with nine knots at deciles (filled circles) and 95\% CIs (open circles) in patients with type 2 diabetes and RI. The relationship between SBP as a continuous variable and the square of SBP for analysis of nonlinear relationship in a Cox regression model, adjustment for covariates as in Table 2 and the 6 year CVD $(=\mathrm{CVE})$ rate 


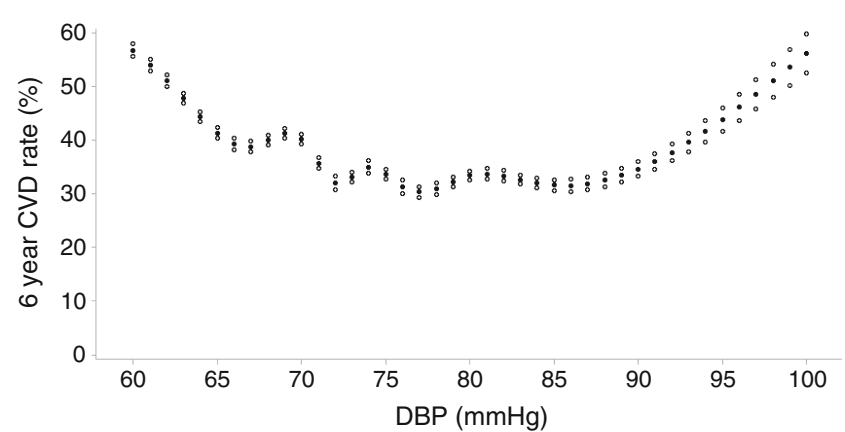

Fig. 2 Spline with nine knots at deciles (filled circles) and 95\% CIs (open circles) in patients with type 2 diabetes and RI. The relationship between the DBP as a continuous variable and the square of DBP for analysis of non-linear relationship in a Cox regression model, adjustment for covariates as in Table 3 , and the 6 year CVD $(=\mathrm{CVE})$ rate

\section{Results}

Clinical and biochemical characteristics at baseline The mean overall eGFR in patients with RI $(N=33,356)$ was $48.2 \pm 9.4 \mathrm{ml} \mathrm{min}-1.73 \mathrm{~m}^{-2}$; thus, the majority of patients with RI had stage 3 chronic kidney disease. Seventy per cent of patients with RI were normoalbuminuric and one-third of patients with RI had previous CVD at the beginning of the study (shown in ESM Table 1). Clinical and biochemical characteristics of patients with type 2 diabetes and RI with and without albuminuria at baseline are shown in Table 1. Sixty per cent of patients with albuminuria had microalbuminuria and 40\% had macroalbuminuria. Patients with albuminuria had a significantly longer diabetes duration, worse glycaemic control, higher mean SBP but lower DBP, i.e. a higher pulse pressure (PP), in spite of more antihypertensive treatment compared with normoalbuminuric patients. Patients with albuminuria were more often men and smokers and more often had a history of CVD, CHF and retinopathy at baseline.

Association between SBP and both cardiovascular events and all-cause mortality in patients with RI During the follow-up period, 11,317 (34\%) CVEs were observed, including 7,704 (23\%) episodes of CHD and 2,284 (6.8\%) of stroke. Overall, $10,738(32 \%)$ patients died. The two most common causes of death were CVD (34\%) and malignancies (18\%). Table 2 displays the incidence and HRs of CVEs and all-cause mortality in each group by deciles of overall reported mean SBP. The lowest incidence of CVEs was observed in patients with a SBP of 135-139 mmHg $(n=719,21.7 \%)$. Approximately half of patients with the lowest SBP $(80-120 \mathrm{mmHg}, n=1,526$ [46.0\%], HR 2.3 [95\% CI 2.0, 2.6]) and half of patients with the highest SBP (160-230 $\mathrm{mmHg}, n=1,621$ [48.8\%], HR 3.0 [95\% CI 2.6, 3.3]) had a CVE during the follow-up period. Among patients with CVEs, the highest incidence of CHD was seen in patients with a SBP of $80-120 \mathrm{mmHg}(n=1,211$ [36.5\%], HR 2.6 [95\% CI 2.3, 3.0]) and a SBP of 160 $230 \mathrm{mmHg}(n=1,000$ [31.1\%] HR 2.9 [95\% CI 2.5, 3.4]). The incidence of stroke was highest in patients with a mean SBP of $160-230 \mathrm{mmHg}(n=376$ [11\%], HR 2.6 [95\% CI 2.0, 3.4]). Half (51\%) of patients with a SBP of $80-120 \mathrm{mmHg}$ (HR $2.4[95 \%$ CI $2.1,2.7]$ ) and $46 \%$ of patients with a SBP of

Table 2 Incidence of stroke, CHD, stroke, CVEs and all-cause mortality, and HRs of CVEs and all-cause mortality by deciles of mean SBP ${ }^{\mathrm{a}}$

\begin{tabular}{llllllll}
\hline $\begin{array}{l}\text { SBP interval } \\
(\mathrm{mmHg})\end{array}$ & SBP (mmHg) & Stroke $(n / \%)$ & $\mathrm{CHD}^{\mathrm{b}}(n / \%)$ & $\mathrm{CVE}^{\mathrm{c}}(n / \%)$ & $\mathrm{CVE}^{\mathrm{d}}(\mathrm{HR}[95 \% \mathrm{CI})$ & $\begin{array}{l}\text { All-cause } \\
\text { mortality }(n / \%)\end{array}$ & $\begin{array}{l}\text { All-cause mortality } \\
(\mathrm{HR}[95 \% \mathrm{CI}])\end{array}$ \\
\hline $80-120$ & $114 \pm 7$ & $198 / 6.0$ & $1,211 / 36.5$ & $1,526 / 46.0$ & $2.30(2.03,2.60)$ & $1,686 / 50.8$ & $2.40(2.11,2.73)$ \\
$120-127$ & $124 \pm 2$ & $193 / 5.8$ & $719 / 21.7$ & $1,001 / 30.2$ & $1.37(1.20,1.55)$ & $963 / 29.0$ & $1.40(1.22,1.60)$ \\
$128-131$ & $130 \pm 1$ & $200 / 6.0$ & $808 / 24.3$ & $1,128 / 34.0$ & $1.64(1.44,1.86)$ & $1,057 / 31.8$ & $1.53(1.35,1.76)$ \\
$131-135$ & $134 \pm 1$ & $186 / 5.6$ & $673 / 20.3$ & $956 / 28.8$ & $1.36(1.20,1.55)$ & $837 / 25.2$ & $1.22(1.05,1.41)$ \\
$135-139$ & $137 \pm 1$ & $169 / 5.1$ & $458 / 13.8$ & $719 / 21.7$ & $1($ reference group $)$ & $678 / 20.4$ & $1($ reference group $)$ \\
$139-142$ & $140 \pm 1$ & $250 / 7.5$ & $826 / 24.9$ & $1,201 / 36.2$ & $1.78(1.57,2.02)$ & $1,118 / 33.7$ & $1.61(1.41,1.84)$ \\
$142-146$ & $144 \pm 1$ & $209 / 6.3$ & $662 / 19.7$ & $994 / 30.0$ & $1.39(1.22,1.58)$ & $893 / 26.9$ & $1.23(1.07,1.42)$ \\
$146-151$ & $149 \pm 2$ & $254 / 7.6$ & $718 / 21.6$ & $1,153 / 34.7$ & $1.77(1.56,2.01)$ & $983 / 29.6$ & $1.30(1.13,1.50)$ \\
$151-160$ & $155 \pm 3$ & $249 / 7.5$ & $629 / 18.9$ & $1,018 / 30.7$ & $1.46(1.28,1.66)$ & $987 / 29.6$ & $1.27(1.10,1.46)$ \\
$160-230$ & $169 \pm 10$ & $376 / 11.3$ & $1,000 / 31.1$ & $1,621 / 48.8$ & $2.95(2.62,3.34)$ & $1,536 / 46.3$ & $2.02(1.78,2.30)$ \\
\hline
\end{tabular}

BPs are means \pm SD

${ }^{\text {a }}$ All patients had RI (eGFR $<60 \mathrm{ml} \mathrm{min}^{-1} 1.73 \mathrm{~m}^{-2}$ ); the mean follow-up time was 5.3 years

${ }^{\mathrm{b}}$ Fatal and non-fatal CHD

${ }^{\mathrm{c}}$ Fatal and non-fatal CVE

${ }^{\mathrm{d}} \mathrm{HR}(95 \% \mathrm{CI})$ adjusted for age, diabetes duration, sex, $\mathrm{HbA}_{1 \mathrm{c}}$, BMI, presence/absence of albuminuria, smoking, LDL-cholesterol, triacylglycerol/HDL, history of CVD, previous history of CHF, and antihypertensive and lipid-lowering treatment

${ }^{\mathrm{e}} \mathrm{SBP}$ of $135-139 \mathrm{mmHg}$ was defined as the reference group 
160-230 mmHg (HR 2.0 [95\% CI 1.8, 2.3]) died during the follow-up period. An analysis also adjusting for DBP is shown in ESM Table 2.

When using only the initial reported SBP at baseline instead of the overall reported mean SBP, overall HRs were lower and a significantly higher HR was found only for allcause mortality in the lowest SBP interval $(80-120 \mathrm{mmHg}$, HR 1.25 [95\% CI 1.10, 1.41]; ESM Table 3).

As SBP and/or DBP may change prior to a CVE or death, we performed analyses in which we censored the last reported SBP before a CVE or death (ESM Table 4). In these analyses, we found the highest risks in the lowest and highest SBP intervals of both CVEs and all-cause mortality (in a similar pattern). However, the HRs were lower overall than when mean BPs were used for CVEs (HR 1.2 [95\% CI 1.0, 1.3] vs HR $2.3[95 \%$ CI 2.0, 2.6] for the lowest and HR 1.4 [95\% CI 1.2, 1.5] vs HR 2.9 [95\% CI 2.6, 3.3] for the highest SBP interval) and for all-cause mortality (HR 1.0 [95\% CI $0.96,1.3]$ vs HR 2.4 [95\% CI 2.1, 2.7] for the lowest and HR 1.7 [95\% CI 1.5, 1.9] vs HR 2.0 [95\% CI 1.8, 2.3] for the highest SBP interval).

Unadjusted analyses assessing the relationship of both SBP and DBP with CVE or all-cause mortality show similar patterns, but the magnitudes of risk (i.e. HRs) are higher for all BP groups (data shown in ESM Tables 5 and 6).

When patients with prior CVD and/or CHF were excluded, findings were very similar to those previously described, such that the lowest and highest SBP levels were still associated with the highest risk of CVEs and all-cause mortality (shown in ESM Table 7). It should, however, be noted that the HRs for CVEs and all-cause mortality were somewhat higher overall in the different SBP intervals when comparing patients without prior CVD and/or CHF with all patients.

In addition, stratification for the absence or presence of albuminuria did not alter results substantially (shown in ESM Table 8 and 9). However, it should be noted that the HR for CVE in the lowest SBP group was slightly higher for normoalbuminuric patients than for those with albuminuria (HR 2.4 [95\% CI 2.1, 2.8] vs HR 2.2 [95\% CI 1.8, 2.6], respectively).

Unadjusted analyses of interaction between both SBP and DBP and potential confounders on risk of CVE and all-cause mortality were also performed. These data for are shown in ESM Tables 10 and 11.

The non-linear spline of the 6 year rate of CVEs by mean SBP as a continuous variable is shown in Fig. 1. The risk of CVE was adjusted as described for the Cox model in Table 2. A progressively increased risk of CVEs was seen with both $\mathrm{SBP}<110 \mathrm{mmHg}$ and SBP $>150 \mathrm{mmHg}$. In addition, and interestingly, slight increases in risk of CVEs were found for SBPs of 130 and $140 \mathrm{mmHg}$. These increases in HRs mirrored the results found in the Cox model for the SBP intervals 128131 and $139-142 \mathrm{mmHg}$ in Table 2.
Association between DBP and both cardiovascular events and all-cause mortality in patients with RI Table 3 gives the prevalence and HRs of CVEs and all-cause mortality in each group by DBP. Patients with DBP of $40-63 \mathrm{mmHg}$ and $83-$ $125 \mathrm{mmHg}$ had the highest incidence and risk of CVEs and all-cause mortality: CVEs $(n=1,625$ [49.0\%], HR 2.0 [95\% CI $1.8,2.2])$ and all-cause mortality $(n=1,662[50.1 \%]$, HR 2.0 [95\% CI 1.8, 2.2]), and CVEs ( $n=1,235$ [37\%], HR 2.3 [95\% CI 2.0, 2.5]) and all-cause mortality $(n=1,151$ [35\%], HR 2.3 [95\% CI 2.0, 2.6]), respectively. The highest incidence of CHD occurred in patients with very low DBP (40$63 \mathrm{mmHg}, n=1,197$ [36.1\%], HR 2.1 [95\% CI 1.8, 2.4]) but, as expected, the highest incidence of stroke was found in patients with the highest DBP $(83-125 \mathrm{mmHg}, n=323$ [9.7\%], HR 2.6 [95\% CI 2.0, 3.4]). An additional analysis adjusting for SBP is shown in ESM Table 12.

In a complementary analysis, we censored the last reported DBP before a CVE or death (data presented in ESM Table 13). The highest risks of CVEs and allcause mortality were found in the lowest and highest DBP intervals but the HRs were lower overall compared with using the mean BPs, except for all-cause mortality in the highest DBP interval $(83-125 \mathrm{mmHg}$ ), where the HRs were nearly identical in the two different analyses (HR 2.3 [95\% CI 2.0, 2.6] for both).

The non-linear spline of the 6 year rate of CVEs with mean DBP as a continuous variable is shown in Fig. 2. The risk of CVE was adjusted as described for the Cox model in Table 3. The risk of CVEs increased significantly with a DBP of $<65$ and $>95 \mathrm{mmHg}$. A U-shaped relationship between DBP and CVEs was found, except for DBPs around 70, 75 and $80 \mathrm{mmHg}$. These increases in HRs mirror the results found in the Cox model for the DBP intervals 70-72, 74-76 and 78$80 \mathrm{mmHg}$ shown in Table 3.

Association between mean arterial BP and PP, cardiovascular events and all-cause mortality in patients with $R I$ The highest incidence of CVEs ( $n=1,570$ [47.3\%]) and all-cause mortality $(n=1,668$ [50.3\%]) were seen in the lowest mean arterial pressure (MAP) interval (53-85 mmHg). Using the MAP interval 93-95 $\mathrm{mmHg}$ as a reference group, the highest risk of CVEs (adjusted HR $2.195 \%$ CI 1.9, 2.4) and all-cause mortality (adjusted HR 1.60 [95\% CI 1.4, 1.8]; both $p<0.001$ ) were seen for the highest MAP interval $(107-153 \mathrm{mmHg}$ ), as shown in ESM Table 14.

The highest incidence of $\operatorname{CVD}(n=1,543$ [46.5\%]) and allcause of mortality ( $n=1,517$ [45.7\%]) was seen for the highest PP interval $(85-154 \mathrm{mmHg})$. Using a PP of $61-65 \mathrm{mmHg}$ as the reference group, the lowest PP interval (15-49 $\mathrm{mmHg}$ ) had the highest risk of all-cause mortality (adjusted HR 2.1 [95\% CI 1.8, 2.4]) and the highest PP interval $(85-154 \mathrm{mmHg})$ had the highest risk of CVEs (adjusted HR 2.0 [95\% CI 1.8, 2.2]). When PP was also adjusted for MAP, the HRs for CVEs and 
Table 3 Incidence of stroke, CHD, stroke, CVEs, all-cause mortality and HRs of CVE and all-cause mortality by deciles of mean DBP

\begin{tabular}{llllllll}
\hline $\begin{array}{l}\text { DBP interval } \\
(\mathrm{mmHg})\end{array}$ & $\begin{array}{l}\mathrm{DBP} \\
(\mathrm{mmHg})\end{array}$ & Stroke $(n / \%)$ & $\mathrm{CHD}^{\mathrm{b}}(n / \%)$ & $\mathrm{CVE}^{\mathrm{c}}(n / \%)$ & $\begin{array}{l}\mathrm{CVE}^{\mathrm{d}} \\
(\mathrm{HR}[95 \% \mathrm{CI}])\end{array}$ & $\begin{array}{l}\text { All-cause } \\
\text { mortality }(n / \%)\end{array}$ & $\begin{array}{l}\text { All-cause mortality } \\
(\mathrm{HR}[95 \% \mathrm{CI}])\end{array}$ \\
\hline $40-63$ & $50 \pm 3$ & $226 / 6.8$ & $1,197 / 36.1$ & $1,625 / 49.0$ & $2.0(1.80,2.22)$ & $1,662 / 50.1$ & $2.00(1.78,2.24)$ \\
$63-67$ & $65 \pm 1$ & $168 / 5.1$ & $857 / 25.8$ & $1,141 / 34.4$ & $1.21(1.07,1.37)$ & $1,116 / 33.6$ & $1.21(1.07,1.37)$ \\
$67-70$ & $68 \pm 1$ & $202 / 6.1$ & $658 / 19.8$ & $999 / 30.1$ & $1.15(1.01,1.30)$ & $911 / 27.4$ & $1.14(1.03,1.30)$ \\
$70-72$ & $70 \pm 0.5$ & $246 / 7.4$ & $952 / 28.7$ & $1,349 / 40.6$ & $1.88(1.67,2.11)$ & $1,311 / 39.5$ & $1.88(1.67,2.11)$ \\
$72-74$ & $73 \pm 1$ & $193 / 5.8$ & $575 / 17.3$ & $870 / 26.2$ & $1($ reference group) & $801 / 24.1$ & $1($ reference group) \\
$74-76$ & $75 \pm 0.5$ & $222 / 6.7$ & $726 / 21.9$ & $1,076 / 32.4$ & $1.43(1.26,1.62)$ & $1,007 / 30.3$ & $1.44(1.28,1.63)$ \\
$76-78$ & $77 \pm 1$ & $197 / 5.9$ & $541 / 16.3$ & $837 / 25.2$ & $1.23(1.08,1.40)$ & $706 / 21.3$ & $1.24(1.01,1.41)$ \\
$78-80$ & $79 \pm 1$ & $261 / 7.9$ & $753 / 22.7$ & $1,154 / 34.8$ & $1.78(1.58,2.01)$ & $1,091 / 32.9$ & $1.81(1.60,2.04)$ \\
$80-83$ & $81 \pm 1$ & $246 / 7.4$ & $655 / 19.7$ & $1,031 / 31.1$ & $1.60(1.40,1.80)$ & $982 / 29.6$ & $1.62(1.42,1.82)$ \\
$83-125$ & $88 \pm 4$ & $323 / 9.7$ & $790 / 23.80$ & $1,235 / 37.2$ & $2.26(2.00,2.54)$ & $1,151 / 34.7$ & $2.30(2.03,2.59)$ \\
\hline
\end{tabular}

BPs are means \pm SD

${ }^{a}$ All patients had RI (eGFR $<60 \mathrm{ml} \mathrm{min}^{-1} 1.73 \mathrm{~m}^{-2}$ ); the mean follow-up time was 5.3 years

${ }^{\mathrm{b}}$ Fatal and non-fatal CHD

${ }^{\mathrm{c}}$ Fatal and non-fatal CVD

${ }^{\mathrm{d}} \mathrm{HR}(95 \% \mathrm{CI})$ adjusted for age, diabetes duration, sex, $\mathrm{HbA}_{1 \mathrm{c}}$, BMI, presence/absence of albuminuria, smoking, LDL-cholesterol, triacylglycerol/HDL, history of CVD, previous history of $\mathrm{CHF}$, and antihypertensive and lipid-lowering treatment

${ }^{\mathrm{e}} \mathrm{DBP}$ of $72-74 \mathrm{mmHg}$ was defined as the reference group

all-cause mortality did not change to a major extent. These data are shown in the ESM Tables 15 and 16.

\section{Discussion}

In this nationwide observational study of more than 30,000 patients with type 2 diabetes and RI followed for a median of 5.3 years, we confirm that patients with the lowest and highest mean systolic and diastolic arterial BP intervals are exposed to the highest risks of CVEs and all-cause mortality. A SBP level of 135-139 and a DBP level of 72-74 $\mathrm{mmHg}$ were associated with the lowest risks of CVEs or death in this cohort of patients. A low PP was associated with a high risk of death, but a high PP was associated with an increased risk of a CVE.

Clinical trials have previously shown that a high BP increases the risk of CVEs (especially stroke) and all-cause mortality in patients with type 2 diabetes [23, 24]. However, in the Action to Control Cardiovascular Risk in Diabetes (ACCORD) BP study in patients with nearly to normal renal function, a target SBP of $\leq 120 \mathrm{mmHg}$ did not reduce the risk of major CVE compared with a SBP of $\leq 140 \mathrm{mmHg}$ [10]. Also, in a subgroup analysis of participants with diabetes and coronary artery disease in the International Verapamil SR-Trandolapril (INVEST) study, tight control of SBP $(\leq 130 \mathrm{mmHg}$ ) was not associated with improved cardiovascular outcomes compared with conventional BP control (130$140 \mathrm{mmHg}$ ), and a non-significant increase in the rate of allcause mortality was noted with tight BP control [11]. A J-shaped relationship was found in the Veterans Affairs Diabetes Trial (VADT), in which a higher risk of CVEs was found in patients with SBP $\geq 140 \mathrm{mmHg}$ and DBP $<70 \mathrm{mmHg}$ [13].

Fewer studies have addressed the association between low BP levels and mortality in patients with diabetes and mild to moderate RI, and these studies have reported slightly varying results. In a post hoc analysis of the Irbesartan Diabetic Nephropathy Trial (IDNT), 1,590 patients with type 2 diabetes, albuminuria and mainly mild renal dysfunction, SBP of $<120 \mathrm{mmHg}$ was associated with a higher risk of CV mortality and CVEs. In addition, DBP of $<85 \mathrm{mmHg}$ was associated with a non-significantly higher risk of all-cause mortality and a significantly higher risk of MI but lower risk of stroke [25]. In the Ongoing Telmisartan Alone and in combination with Ramipril Global Endpoint Trial (ONTARGET) trial in which $24 \%$ of all participants had type 2 diabetes, there was no improvement in fatal or non-fatal CVEs, except for stroke, when reducing the SBP below $130 \mathrm{mmHg}$ [26]. Similar results were later found in the Aliskiren Trial in Type 2 Diabetes Using Cardio-Renal Endpoints (ALTITUDE) and the Veterans Affairs Nephropathy in Diabetes (VA NEPHROND) trials: findings showed no benefit with respect to mortality or CVEs and that intensive treatment with dual reninangiotensin-aldosterone system (RAAS) blockade even may even be harmful. The effects of BP lowering and/or RAAS blockade are therefore still under debate [27, 28].

A J-shaped relationship between BP and CVEs or mortality has been discussed in several reports and was, for example, found in the VADT where a higher risk of CVEs was seen in patients with SBP $\geq 140 \mathrm{mmHg}$ and $\mathrm{DBP}<70 \mathrm{mmHg}$ [13], 
indicating that both too high $\mathrm{BP}$ and too low $\mathrm{BP}$ are associated with an increased risk of CVEs and mortality [13]. In patients with RI, a low BP is likely to be a marker of either pre-existing CVD (including CHF) or treatment of these conditions with agents with BP-lowering properties, or both [29, 30].

In the present study, a more U-shaped relationship was observed between mean BP levels and CVEs despite the fact that only $15 \%$ of participants had a history of CHF at baseline and that patients with an eGFR of $<15 \mathrm{ml} \mathrm{min}^{-1} 1.73 \mathrm{~m}^{-2}$ were excluded. The mean eGFR was $48 \pm 9 \mathrm{ml} \mathrm{min}^{-1} 1.73 \mathrm{~m}^{-2}$; thus, most patients had only mild to moderate RI. Even so, the number of cardiovascular events and deaths during the follow-up period was very high (CVEs 34\%, all-cause mortality $32 \%$ ) compared with previous studies [31, 32]. When we omitted/censored the last reported BP before a CVE or death, a similar U-shaped pattern was found but the risk estimates were somewhat lower than when using the mean of all BP measurements reported during follow-up. One potential explanation for this U-shaped relationship may be that in this study we divided patients into BP deciles and not according to static BP values, as has been done in other studies.

The risk of CVEs and all-cause mortality was also significantly higher for the lowest and highest MAP intervals (53-85 and $107-153 \mathrm{mmHg}$ ). MAP is highly correlated with SBP and DBP, and its value as a better predictor of CVD than SBP and/ or DBP has therefore been questioned [33, 34].

We also evaluated the association of PP with both CVEs and all-cause mortality. A high PP is mainly related to increased stiffness in the large arteries, as opposed to a low PP, which is often a marker of low SBP or, rarely, of isolated diastolic hypertension. PP is recognised as a predictor of CVEs and, in agreement with other studies, our result also showed that the risk of CVEs or all-cause mortality is greater for the highest PP interval $(85-154 \mathrm{mmHg})[35,36]$. The high risk of death for the lowest PP interval $(15-49 \mathrm{mmHg})$ is likely to be a consequence of low SBP. In this study, the relationship between PP and CVEs was relatively weak and became even weaker when PP was adjusted for MAP. This is consistent with the results of previous studies on the relationships between different BP indices and cardiovascular outcomes [37-39].

Albuminuria was found only in $30 \%$ of patients with RI at baseline, in line with previous studies in which the majority of patients with type 2 diabetes and RI were found to be normoalbuminuric [40-42]. Both albuminuria and RI are independent risk factors for CVEs and all-cause mortality in type 2 diabetes, with albuminuria being the strongest risk factor and relevant at all levels of renal function. However, in normoalbuminuric patients, a slight reduction in renal function is an important predictor of CVEs and all-cause mortality [43]. The observation that the majority of patients were normoalbuminuric is of interest because treatment guidelines generally recommend even lower BP targets in patients with albuminuria [16]. Although albuminuria has been recognised as a risk factor for CVEs and mortality, adjustments for the presence of albuminuria did not markedly alter the results in the present study [44]. However, an interesting finding of this study was that the risk of CVEs in the lowest BP interval was actually slightly higher for normoalbuminuric patients than for those with albuminuria.

The major strengths of this cohort study are the nationwide scale, large number of patients and the many person-years of observation and number of events. We included patients who received routine treatment according to national guidelines in both primary and secondary care, supporting high external validity and generalisability of our findings to other type 2 diabetes populations. In this study, participants were divided into deciles to reflect the true variation in BP readings, instead of using the traditional BP cutoffs. However, there are some limitations to this study. Since this is an observational study, a cause-effect relationship cannot be established. Moreover, the data were reported by different medical centres and laboratories, which may have slightly affected the accuracy of the data. Only clinical BP measurements were used in this study and not ambulatory BPs, which could be an even better predictor of cardiovascular risk [45, 46]. However, less than $20 \%$ of participants had only one reported BP measurement during the study period. It is also plausible that the true BP value for some patients in these BP intervals were higher when measured at the centres, but rounded downwards when reporting in order to comply with national treatment guidelines. This, we think, might explain the slight variation in risk seen for the lower BP intervals, i.e. participants with an SBP interval of $139-142 \mathrm{mmHg}$ or a DBP interval of 78$80 \mathrm{mmHg}$ were shown to exhibit an increased risk of CVE and all-cause mortality compared with those with either a lower or a higher achieved BP. Thus, if a high risk of $\mathrm{CVD}$ is attributed to false low $\mathrm{BP}$ readings and a CVE occurs, then this is likely to occur. Further standardisation of BP measurements using automated methods could possibly reduce such bias.

In conclusion, the risk of cardiovascular disease and allcause mortality was very high in patients with type 2 diabetes and RI in clinical practice, and increased significantly with both high and low SBP and DBP in a U-shaped manner. A SBP of $135-139 \mathrm{mmHg}$ and a DBP of $72-74 \mathrm{mmHg}$ were associated with the lowest risks of CVE and all-cause mortality.

Acknowledgements We would like to thank the regional NDR coordinators and all participating nurses, physicians and other staff who contributed to the NDR. Most of all, we would like to thank the patients who support the NDR, both individually and collectively through their patient organisation, and the Swedish Diabetes Federation and the Swedish Board of Health and Welfare. We would also especially like to thank M. Miftaraj at the Center of Registers in Region Västra Götaland, Gothenburg, Sweden, for her statistical expertise. 
Some of the data in this paper has previously been presented as an abstract at the 51st ERA-EDTA congress, Amsterdam, the Netherlands, 31 May-3 June 2014.

Funding Supported by grants from the Swedish government (under the Avtal om Läkarutbildning och medicinsk Forskning [agreement for medical education and research]). This work was also supported by grants for research and medical education from Skaraborgs Hospital, Skövde, Sweden (project number 53340).

Duality of interest MKS is currently employed by Amgen AB. The other authors declare that there is no duality of interest associated with their contribution to this manuscript.

Contribution statement All authors researched the data; MP, A-MS and HA performed the statistical analyses; HA and MKS drafted the manuscript; and all authors reviewed and contributed to the manuscript and thereafter approved the version to be published. MKS is the guarantor of this work.

\section{References}

1. Turner RC, Holman RR (1996) The UK Prospective Diabetes Study. UK Prospective Diabetes Study Group. Ann Med 28:439-444

2. Collins R, Peto R, MacMahon S et al (1990) Blood pressure, stroke, and coronary heart disease. Part 2, Short-term reductions in blood pressure: overview of randomised drug trials in their epidemiological context. Lancet 335:827-838

3. Chobanian AV, Bakris GL, Black HR et al (2003) The Seventh Report of the Joint National Committee on Prevention, Detection, Evaluation, and Treatment of High Blood Pressure: the JNC 7 report. JAMA 289:2560-2572

4. O'Donnell MJ, Xavier D, Liu L et al (2010) Risk factors for ischaemic and intracerebral haemorrhagic stroke in 22 countries (the INTE RSTROKE study): a case-control study. Lancet 376:112-123

5. Lewington S, Clarke R, Qizilbash N, Peto R, Collins R, Prospective Studies C (2002) Age-specific relevance of usual blood pressure to vascular mortality: a meta-analysis of individual data for one million adults in 61 prospective studies. Lancet 360:1903-1913

6. Savoia C, Schiffrin EL (2007) Vascular inflammation in hypertension and diabetes: molecular mechanisms and therapeutic interventions. Clin Sci 112:375-384

7. Cederholm J, Gudbjornsdottir S, Eliasson B et al (2012) Blood pressure and risk of cardiovascular diseases in type 2 diabetes: further findings from the Swedish National Diabetes Register (NDR-BP II). J Hypertens 30:2020-2030

8. (1998) Tight blood pressure control and risk of macrovascular and microvascular complications in type 2 diabetes: UKPDS 38. UK Prospective Diabetes Study Group. BMJ 317: 703-713

9. Patel A, Group AC, MacMahon S et al (2007) Effects of a fixed combination of perindopril and indapamide on macrovascular and microvascular outcomes in patients with type 2 diabetes mellitus (the ADVANCE trial): a randomised controlled trial. Lancet 370: $829-840$

10. Mancia G (2010) Effects of intensive blood pressure control in the management of patients with type 2 diabetes mellitus in the Action to Control Cardiovascular Risk in Diabetes (ACCORD) trial. Circulation 122:847-849

11. Cooper-DeHoff RM, Gong Y, Handberg EM et al (2010) Tight blood pressure control and cardiovascular outcomes among hypertensive patients with diabetes and coronary artery disease. JAMA 304:61-68
12. Weber MA, Bakris GL, Hester A et al (2013) Systolic blood pressure and cardiovascular outcomes during treatment of hypertension. Am J Med 126:501-508

13. Anderson RJ, Bahn GD, Moritz TE et al (2011) Blood pressure and cardiovascular disease risk in the Veterans Affairs Diabetes Trial. Diabetes Care 34:34-38

14. Dorresteijn JA, van der Graaf Y, Spiering W et al (2012) Relation between blood pressure and vascular events and mortality in patients with manifest vascular disease: J-curve revisited. Hypertension 59: 14-21

15. Hayes W (2013) 2013 American Diabetes Association update: treatment of hypertension in patients with diabetes. S D Med 66:236-237

16. Taler SJ, Agarwal R, Bakris GL et al (2013) KDOQI US commentary on the 2012 KDIGO clinical practice guideline for management of blood pressure in CKD. Am J Kidney Dis 62:201-213

17. Levey AS, Bosch JP, Lewis JB, Greene T, Rogers N, Roth D (1999) A more accurate method to estimate glomerular filtration rate from serum creatinine: a new prediction equation. Modification of Diet in Renal Disease Study Group. Ann Intern Med 130:461-470

18. Zethelius B, Eliasson B, Eeg-Olofsson K et al (2011) A new model for 5-year risk of cardiovascular disease in type 2 diabetes, from the Swedish National Diabetes Register (NDR). Diabetes Res Clin Pract 93:276-284

19. Ekstrom N, Cederholm J, Zethelius B et al (2013) Aspirin treatment and risk of first incident cardiovascular diseases in patients with type 2 diabetes: an observational study from the Swedish National Diabetes Register. BMJ Open 3:e02688

20. Ludvigsson JF, Andersson E, Ekbom A et al (2011) External review and validation of the Swedish national inpatient register. BMC Public Health 11:450

21. Tunstall-Pedoe H, Kuulasmaa K, Amouyel P, Arveiler D, Rajakangas AM, Pajak A (1994) Myocardial infarction and coronary deaths in the World Health Organization MONICA Project. Registration procedures, event rates, and case-fatality rates in 38 populations from 21 countries in four continents. Circulation 90:583-612

22. Cederholm J, Zethelius B, Eliasson B, Gudbjornsdottir S, Nilsson PM (2012) Different methods to present the effect of blood pressure on cardiovascular diseases by Cox regression. J Hypertens 30: 235-237

23. Adler AI, Stratton IM, Neil HA et al (2000) Association of systolic blood pressure with macrovascular and microvascular complications of type 2 diabetes (UKPDS 36): prospective observational study. BMJ 321:412-419

24. Schrier RW, Estacio RO, Mehler PS, Hiatt WR (2007) Appropriate blood pressure control in hypertensive and normotensive type 2 diabetes mellitus: a summary of the $\mathrm{ABCD}$ trial. Nature clinical practice. Nephrology 3:428-438

25. Berl T, Hunsicker LG, Lewis JB et al (2005) Impact of achieved blood pressure on cardiovascular outcomes in the Irbesartan Diabetic Nephropathy Trial. J Am Soc Nephrol 16:2170-2179

26. Mancia G, Schumacher H, Redon J et al (2011) Blood pressure targets recommended by guidelines and incidence of cardiovascular and renal events in the Ongoing Telmisartan Alone and in Combination with Ramipril Global Endpoint Trial (ONTARGET). Circulation 124:1727-1736

27. Parving HH, Brenner BM, McMurray JJ et al (2012) Cardiorenal end points in a trial of aliskiren for type 2 diabetes. N Engl J Med 367: 2204-2213

28. Fried LF, Emanuele N, Zhang JH et al (2013) Combined angiotensin inhibition for the treatment of diabetic nephropathy. N Engl J Med 369:1892-1903

29. Kovesdy CP, Trivedi BK, Kalantar-Zadeh K, Anderson JE (2006) Association of low blood pressure with increased mortality in patients with moderate to severe chronic kidney disease. Nephrol Dial Transplant 21:1257-1262 
30. Busby WJ, Campbell AJ, Robertson MC (1994) Is low blood pressure in elderly people just a consequence of heart disease and frailty? Age Ageing 23:69-74

31. Vepsalainen T, Laakso M, Kantola I, Lehto S, Juutilainen A, Ronnemaa T (2012) Proteinuria modifies the effect of systolic blood pressure on total and cardiovascular disease mortality in patients with type 2 diabetes. J Intern Med 272:611-619

32. Kovesdy CP, Bleyer AJ, Molnar MZ et al (2013) Blood pressure and mortality in U.S. veterans with chronic kidney disease: a cohort study. Ann Intern Med 159:233-242

33. Sesso HD, Stampfer MJ, Rosner B et al (2000) Systolic and diastolic blood pressure, pulse pressure, and mean arterial pressure as predictors of cardiovascular disease risk in men. Hypertension 36:801-807

34. Hadaegh F, Shafiee G, Hatami M, Azizi F (2012) Systolic and diastolic blood pressure, mean arterial pressure and pulse pressure for prediction of cardiovascular events and mortality in a Middle Eastern population. Blood Press 21:12-18

35. Franklin SS, Khan SA, Wong ND, Larson MG, Levy D (1999) Is pulse pressure useful in predicting risk for coronary heart Disease? The Framingham heart study. Circulation 100:354-360

36. Assmann G, Cullen P, Evers T, Petzinna D, Schulte H (2005) Importance of arterial pulse pressure as a predictor of coronary heart disease risk in PROCAM. Eur Heart J 26:2120-2126

37. Miura K, Nakagawa H, Ohashi Y et al (2009) Four blood pressure indexes and the risk of stroke and myocardial infarction in Japanese men and women: a meta-analysis of 16 cohort studies. Circulation 119:1892-1898

38. Domanski M, Mitchell G, Pfeffer M et al (2002) Pulse pressure and cardiovascular disease-related mortality: follow-up study of the Multiple Risk Factor Intervention Trial (MRFIT). JAMA 287:2677-2683
39. Glynn RJ, Chae CU, Guralnik JM, Taylor JO, Hennekens CH (2000) Pulse pressure and mortality in older people. Arch Intern Med 160: 2765-2772

40. Rigalleau V, Lasseur C, Raffaitin C et al (2007) Normoalbuminuric renal-insufficient diabetic patients: a lower-risk group. Diabetes Care 30:2034-2039

41. Penno G, Solini A, Bonora E et al (2011) Clinical significance of nonalbuminuric renal impairment in type 2 diabetes. J Hypertens 29: 1802-1809

42. Afghahi H, Miftaraj M, Svensson AM et al (2013) Ongoing treatment with renin-angiotensin-aldosterone-blocking agents does not predict normoalbuminuric renal impairment in a general type 2 diabetes population. J Diabetes Complicat 27:229-234

43. Svensson MK, Cederholm J, Eliasson B, Zethelius B, Gudbjornsdottir S, Swedish National Diabetes R (2013) Albuminuria and renal function as predictors of cardiovascular events and mortality in a general population of patients with type 2 diabetes: a nationwide observational study from the Swedish National Diabetes Register. DiabVasc Dis Res 10:520-529

44. Gerstein HC, Mann JF, Yi Q et al (2001) Albuminuria and risk of cardiovascular events, death, and heart failure in diabetic and nondiabetic individuals. JAMA 286:421-426

45. Eguchi K, Pickering TG, Hoshide S et al (2008) Ambulatory blood pressure is a better marker than clinic blood pressure in predicting cardiovascular events in patients with/without type 2 diabetes. Am J Hypertens 21:443-450

46. Dolan E, Stanton A, Thijs L et al (2005) Superiority of ambulatory over clinic blood pressure measurement in predicting mortality: the Dublin outcome study. Hypertension 46:156-161 